\title{
Tests for intrinsicness tested
}

\author{
Kelvin J. McQueen ${ }^{1}$ - René van Woudenberg ${ }^{1}$
}

Published online: 22 February 2016

(C) The Author(s) 2016. This article is published with open access at Springerlink.com

\begin{abstract}
Various tests have been proposed as helps to identify intrinsic properties. This paper compares three prominent tests (we call them Perfect Duplication, Real Change, and Lonely) and shows that they fail to pass adequate verdicts on a set of three properties. The paper examines whether improved versions of the tests can reduce or remove these negative outcomes. We reach the sceptical conclusion that whereas some of the tests must be discarded as inadequate because they don't yield definite results, the remaining tests depend for their application on the details of fundamental particle physics so much so that they cannot be relied upon.
\end{abstract}

Keywords Intrinsic $\cdot$ Extrinsic $\cdot$ Relational $\cdot$ Properties $\cdot$ Entanglement · Nonseparability

Properties have often been held to fall in neat categories: they are necessary or contingent, essential or accidental, primary or secondary, dispositional or categorical, intrinsic or extrinsic, such or so. This is the broad picture. But the devil is in the usual spot. The detail that is the topic of this paper concerns the category of intrinsic properties, and the problem it discusses is how to distinguish properties that are intrinsic from those that are not. Very roughly, an intrinsic property is a property that 'really belongs' to the item having it. This characterization, however, isn't particularly helpful. Does the property of being the queen of the UK 'really belong' to Elisabeth II? Is being the Evening Star a property that 'really belongs' to the Morning Star? That is not so clear. In the literature, therefore, a number of explicitly

Kelvin J. McQueen

klvnmcqn@gmail.com

1 Faculty of Humanities (Philosophy), VU University Amsterdam, De Boelelaan 1105, $1081 \mathrm{HV}$ Amsterdam, The Netherlands 
so called 'tests' have been proposed as helps. They are introduced in Sect. 1. If the tests yield determinate and converging results, this evidences that they adequately pick out intrinsic properties. As we argue in Sect. 2, however, these tests give neither converging nor determinate results on a set of three properties. The remainder of the paper argues that there is no way out and defends a sceptical conclusion: the only workable tests for intrinsicness cannot be performed without first knowing the fundamental nature of reality-knowledge that we, as a matter of fact, don't have.

We begin with two preliminaries. Firstly, we focus on tests for intrinsicness as they can be found in the literature. ${ }^{1}$ Perhaps more tests can be devised, but these are the ones that actually have been devised. The hallmark of a test is that it is a recipe, it tells you what to do when you want to figure out something-in this case: it tells you what to do when you want to figure out whether a property is intrinsic or not. In this respect tests for intrinsicness differ from analyses of intrinsicness. Analyses aren't recipes - they don't tell you what to do when you want to figure out whether something is an $X$; analyses aim to tell you what it is for something to be an $\mathrm{X}$, they aim to specify the nature of Xs. The tests for intrinsicness that we are going to discuss, then, aim to help us to tell intrinsic properties from non-intrinsic properties; they are proposed as epistemic helps for inquirers, but they don't aim to elucidate the underlying nature of intrinsic properties. There may be interesting relations between tests and analyses, but that is not something we propose to explore in this paper.

At this point it will be appropriate to indicate, albeit provisionally, when a test for intrinsicness is a successful test. As we will be thinking of this matter, a test $\mathrm{T}$ for intrinsicness is a successful test provided (a) $\mathrm{T}$ gives definite results for many, but perhaps not all, properties; (b) T's results are intuitively satisfying; and (c) T's results aren't in conflict with other tests that satisfy (a) and (b).

Our second preliminary is this. It has recently been argued (Figdor 2008), that a distinction should be made between intrinsic/extrinsic on the one hand, and intrinsically/extrinsically on the other. The first is a distinction between properties, the second a distinction between ways in which particulars have properties. Figdor argues that these distinctions do not overlap, so that an extrinsic property can be had intrinsically (her example is being witty), and an intrinsic property can be had extrinsically (her example is being square). This entails that one and the same property can be had intrinsically by one thing, but extrinsically by another. We describe the tests as being directed at the intrinsic/extrinsic distinction. But since our toy set of properties are illustrated in terms of specific instantiations of those properties, our discussion also extends naturally to the intrinsically/extrinsically distinction.

\footnotetext{
1 Apart from the three tests that we will discuss in Sect. 1, there is one further test that can be found in the literature that has been named 'test for intrinsicness' but that we won't discuss because the notion of 'intrinsicness' at play there is such that what intuitively seem to be 'relational' properties (and so the supposed complements of intrinsic properties) on that test also qualify as 'intrinsic'. This test is described in Searle (1995:11-12), and stages an opposition between intrinsic and observer-relative properties.
} 


\section{Tests for intrinsicness}

A first test involves an imaginary machine that produces perfect atom-for-atom duplicates of originals. This test is inspired by David Lewis's remark that "if something has an intrinsic property then so does any perfect duplicate of that thing, whereas duplicates situated in different surroundings will differ in their extrinsic properties." (Lewis 1983: 112) ${ }^{2}$ Peter van Inwagen has described the test as follows: "If a property of the original is intrinsic, it will be reproduced by the machine; that is, it will be a property of the duplicate. Or, what is the same thing, if a property is not reproduced by the machine, it is not intrinsic but relational." (van Inwagen 2009: 40-1) then, opposes intrinsic to relational properties. However, since some properties are arguably ${ }^{3}$ both intrinsic and relational, e.g. having longer arms than legs, and having a proper part, we prefer to say that intrinsic properties oppose extrinsic properties (where 'extrinsic' just means 'not intrinsic'). The test that Van Inwagen writes about can therefore be put as follows:

Perfect Duplication. If property $\mathrm{P}$ of object $\mathrm{O}$ gets copied by the perfect duplication machine, $\mathrm{P}$ is an intrinsic property of $\mathrm{O}$; and if the perfect duplication machine does not copy $\mathrm{P}, \mathrm{P}$ is an extrinsic property of $\mathrm{O}$.

Examples that have been adduced to illustrate the cogency of this test involve the Queen of England. The colour of the Queen's eyes will be copied by the machinehence colour is an intrinsic property. But only the Queen, not her replica, will be the crowned head of the United Kingdom-hence being the crowned head of the United Kingdom is extrinsic.

Perfect Duplication, as formulated, is not entirely satisfactory. Take for instance the property of having the same colour as the object at the entrance of the machine. That property, says van Inwagen, gets copied, and so, by this test, is intrinsic. But this, he avers, is quite obviously wrong; it is relational. He therefore holds that this test is "useful, but fallible", a good rule of thumb-for two reasons. First, failure is conclusive: a property that fails the test has to be relational. Second, the relational properties that pass the test are rather contrived (van Inwagen 2009: 34).

A second test involves the notion of a 'real change' as opposed to a 'mere Cambridge change'. This distinction is due to Geach (1969: 71-2) who introduced it in his discussion of an account of what it takes for an object to undergo change- the account being that an object has changed just in case it has lost (or gained) some property. Geach has argued that this account cannot be correct. For consider, he urges, when Socrates becomes shorter than Theaetetus simply by virtue of the latter's growth. Then Socrates acquires a new property, viz. the property of being shorter than Theaetetus, and on the account under consideration this means that Socrates changes. But, Geach muses, this change is not a real change in Socrates; it

\footnotetext{
${ }^{2}$ For an in-depth discussion and defense of Lewis' account of intrinsic properties, see Sider (1996). Sider states Lewis's analysis of 'intrinsic' as follows: Property $\mathrm{P}$ is intrinsic iff for any possible objects $x$ and $y$, if $x$ and $y$ are duplicates then $x$ has P iff $y$ has $\mathrm{P}$ (Sider 1996: 2). The duplication machine is intended to help one determine whether a property satisfies this definition.

3 See Weatherson and Marshall (2013): Sect. 2.1.
} 
is a real change in Theaetetus. The change in Socrates is a 'mere Cambridge change'. ${ }^{4}$

The second test, as described by Peter van Inwagen, involves the notion of 'real change' (and hence, if only implicitly, the real change/Cambridge change distinction): "if the gain or loss of a property would be a real change in a thing, then that property is intrinsic; otherwise it is extrinsic." 5 So the proposed test is:

Real Change. If object O's loss or gain of property $\mathrm{P}$ would constitute a real change in $\mathrm{O}, \mathrm{P}$ is intrinsic; and if $\mathrm{O}$ 's loss or gain of $\mathrm{P}$ would not constitute a real change in $\mathrm{O}, \mathrm{P}$ is extrinsic.

Examples that have been offered to illustrate the cogency of this test include mass and spatial positions. If a thing loses the property of having a mass of 10 kilograms, the loss would constitute a real change in the thing-hence the property is intrinsic. But if a thing loses the property of being located fifty miles north of a burning barn, the loss would not constitute a real change in the thing-hence it is extrinsic. In this latter case, the loss constitutes a 'mere Cambridge change', i.e. a change that isn't really a change of the object.

A third test, suggested by David Lewis in his discussion of a suggestion by Jaegwon Kim, who was in turn discussing a suggestion by Roderick Chisholm (Lewis 1983: 111-2), is that property $\mathrm{P}$ is intrinsic to object $\mathrm{O}$ provided $\mathrm{O}$ would continue to have $\mathrm{P}$, even if no other thing besides $\mathrm{O}$ were to exist; and it is extrinsic provided $\mathrm{O}$ would lose $\mathrm{P}$ if no other thing besides $\mathrm{O}$ were to exist. This suggestion contains the seeds of a test, to be performed by a thought experiment, viz. to imaginatively remove all contingent objects other than $\mathrm{O}$ from the actual world, and then see what happens to that object's properties:

Lonely. If an object $\mathrm{O}$ in the actual world has property $\mathrm{P}$ and all contingent objects other than $\mathrm{O}$ are imaginatively removed from the world (i.e. "is made lonely"), then if $\mathrm{O}$ retains $\mathrm{P}$, then $\mathrm{P}$ is intrinsic, and if $\mathrm{O}$ loses $\mathrm{P}$, then $\mathrm{P}$ is extrinsic. ${ }^{6}$

Consider the property of being in the vicinity of Trafalgar Square. If you have that property but are imaginatively made lonely, you lose it. Hence that property is extrinsic. But when you are imaginatively made lonely, you remain single headed. Hence, by Lonely, being single headed is an intrinsic property of yours.

These are the tests that we shall be considering. After showing that they are inadequate, we will consider certain possible fixes, and we will argue that no such fixes are plausible.

\footnotetext{
${ }^{4}$ For a discussion of so-called Cambridge properties, see Francescotti (1999).

5 van Inwagen (2009): 40-1. A related version of this test is offered by Stump and Kretzman who aver that "change in x's extrinsic properties can occur without a change in $x$, while a change in $x$ 's intrinsic properties is as such a change in $\mathrm{x}$ " (Stump and Kretzman 1985).

6 This test is in fact closer to Vallentyne's thoughts on intrinsicness than to Lewis's. See (Vallentyne 1997; Marshall 2013).
} 


\section{Tests for intrinsicness compared}

Do these tests yield the same results? Do they yield determinate results? This section answers both questions in the negative by applying the tests to the following toy set of properties:

[a] being a one-euro coin

[b] being in Amsterdam

[c] wearing a golden ring

\subsection{Tests applied to [a]: being a one-euro coin}

Some pieces of metal have the property of being a one-euro coin $(=[\mathrm{a}])$. A perfect duplicate of such a piece of metal will be indistinguishable from the original. Still, the duplicate may not be a real one-euro coin, for in order to qualify as such it must be issued by a legal authority, which it may not. And if it isn't, it's a forgery and not really a one-euro coin. Whether the duplicate has [a], then, depends on who operates the machine. By Perfect Duplication [a] is intrinsic if the machine is legally operated. But if the machine is not legally operated, the duplicate is not really a oneeuro coin, hence it doesn't have [a], which means that [a] is not copied, which means that $[\mathrm{a}]$ is extrinsic.

If a piece of metal loses [a] because it is melted, then the change is a real change of the piece of metal-a change that entails the loss of [a]. By Real Change, then, [a] is intrinsic to the piece of metal. But if that piece of metal loses [a] because a new law has been issued ruling that one-euro coins are no longer legal tender, then the piece of metal does not undergo a real change, only a change that can be compared with Socrates when he becomes shorter than Theaetetus due to the latter's growth. Hence by Real Change [a] is extrinsic.

Applying Lonely, it is reasonable to suppose that since there are no mints in the lonely world, there is no money, hence the piece of metal is not money; hence [a] is extrinsic.

\subsection{Tests applied to [b]: being in Amsterdam}

Suppose a particular molecule is in Amsterdam, and the duplication machine is also in Amsterdam. Then both the original molecule and its duplicate will have [b]: the property of being in Amsterdam. Hence by Perfect Duplication [b] is intrinsic. But if we suppose that the entrance of the machine is in Amsterdam, but not its exit, then the original will have [b] but the duplicate won't-in which case Perfect Duplication entails that [b] is extrinsic. Hence, whether or not [b] is intrinsic depends on the location of the entrance and exit of the machine.

\subsubsection{Regarding real change}

If the molecule loses [b] because Earth rotates relative to the molecule, then the molecule does not undergo a real change, only a change that can be compared with 
Socrates when he becomes shorter than Theaetetus due to the latter's growth. Hence by Real Change [b] is extrinsic. However, if the molecule loses [b] because the size of its atomic constituents, together with their relative distance, are increased so much so that the molecule can no longer be said to be in Amsterdam, then the molecule does undergo a real change. By Real Change, then, [b] is intrinsic to the molecule.

Would the molecule, made lonely, still be in Amsterdam? No it would not, there being no Amsterdam in that lonely world. Hence, by Lonely, [b] is extrinsic.

\subsection{Tests applied to [c]: wearing a golden ring}

If the Queen wears a golden ring, and is fed into the duplication machine, will her duplicate wear a ring too? This depends on whether we feed the ring, along with the Queen, into the duplication machine. If we feed Queen-with-ring to the machine, the duplicate will wear a ring too, which means that [c] comes out as intrinsic. But if we feed Queen-minus-ring to the machine, the duplicate won't have [c], which means [c] is not intrinsic. So, Perfect Duplication doesn't give a definite answer with respect to [c] until this test allows us to determine, without appeal to 'intrinsic', whether the Queen should retain her ring or not when entering the machine. But it is unclear how, or whether, the test can allow so much. This is a crucial point that we shall return to in detail.

If the Queen unknowingly loses her ring down the drain while showering, there appears to be no real change in the Queen, or at least no more of a change than her unknowingly losing a strand of hair. But if the Queen loses her ring due to an unfortunate accident while chopping vegetables in the kitchen, it would appear we have a real change in the Queen, at least insofar as losing one's finger constitutes a real change to a person. Once again, Real Change gives conflicting results.

Lonely suffers a similar problem to Perfect Duplication: when we put the Queen into the lonely world do we just take her as she is (jewellery and all), or must we 'prepare' her in a way that involves removing the ring? The former yields intrinsic with respect to [c], while the latter yields extrinsic. The test as formulated so far does not have the resources to yield a determinate answer.

The following table summarizes the discussion of this section:

$\begin{array}{clll}\begin{array}{c}\text { [a] Being a } \\ \text { one-euro } \\ \text { coin }\end{array} & \begin{array}{c}\text { Perfect Duplication } \\ \text { Depends on legal } \\ \text { status of machine }\end{array} & \begin{array}{c}\text { Real Change } \\ \text { Depends on the change } \\ \text { (melting vs. legal ruling) }\end{array} & \begin{array}{l}\text { Lonely } \\ \text { Extrinsic }\end{array} \\ \begin{array}{c}\text { [b] Being in } \\ \text { Amsterdam }\end{array} & \begin{array}{c}\text { Depends on the } \\ \text { location of the } \\ \text { machine }\end{array} & \begin{array}{c}\text { Depends on the change } \\ \text { (rotation vs. } \\ \text { enlargement) }\end{array} & \text { Extrinsic } \\ \begin{array}{c}\text { [c] Wearing } \\ \text { a golden } \\ \text { ring }\end{array} & \begin{array}{c}\text { Depends on what's } \\ \text { fed to the machine }\end{array} & \begin{array}{c}\text { Depends on the change } \\ \text { (shower vs. kitchen } \\ \text { accident) }\end{array} & \begin{array}{c}\text { Depends on } \\ \text { what's made } \\ \text { lonely }\end{array}\end{array}$


The discussion so far enables us to make three embarrassing observations:

1. Typically both of the alternative and allegedly mutually exclusive verdicts can be reached (Perfect Duplication on [a], [b] and [c]; Real Change on [a], [b] and [c], Lonely on [c]).

2. No one test gives the same series of verdicts on [a]-[e] as any of the other tests.

3. None of the properties are tested by all the tests as intrinsic, and none are tested by all the tests as non-intrinsic.

Can we reduce the embarrassment? Let us begin by reconsidering Real Change.

\section{Farewell real change}

The formulations of our three tests all had something in common: they require us to test whether a given object retains a property (or undergoes a real change) in a given world. This, however, means that the features of those worlds may impact the results of the tests in undesired ways. To forestall such undesired influences we can, perhaps, improve the tests by making them more general. In the case of Real Change it seems we just need to ensure that a real change is guaranteed by the loss or gain of an intrinsic property:

Real Change*. In all possible worlds in which object $\mathrm{O}$ loses or gains property $\mathrm{P}$, if $\mathrm{O}$ 's loss or gain of property $\mathrm{P}$ constitutes a real change in $\mathrm{O}, \mathrm{P}$ is intrinsic; otherwise $\mathrm{P}$ is extrinsic.

This increase in modal strength leads to sensible determinate results for our test cases. And it is easy to see why: finding just one world in which $\mathrm{O}$ does not undergo a real change despite losing P (e.g. a world in which the Queen loses her ring in the shower) is sufficient to guarantee a determinate test result: extrinsic. Note that Perfect Duplication admits a similar fix:

Perfect Duplication*. In all possible worlds in which object $\mathrm{O}$ has property $\mathrm{P}$ and $\mathrm{O}$ is fed into a duplication machine, if $\mathrm{P}$ is retained by $\mathrm{O}$ 's duplicate, $\mathrm{P}$ is an intrinsic property of $\mathrm{O}$; otherwise $\mathrm{P}$ is extrinsic.

Consider [b], the property of being in Amsterdam. Whether that property comes out as intrinsic or not, we suggested, depends on where the machine outputs O's duplicate. But only in some possible worlds will the machine output O's duplicate in Amsterdam. In other worlds, it will output O's duplicate in New Zealand. Since there are worlds in which O's duplicate fails to possess [b], [b] is extrinsic-in line with our intuitions. Clearly, a similar point can be made about [a]. However, as we shall see, $[\mathrm{c}]$ is going to point to deeper difficulties. We will return to this, once we have said farewell to Real Change*.

Let us recall the piece of metal which has [a], the property of being a one-euro coin. We asked whether that piece of metal's loss of [a] would constitute a real change in it. What our discussion brought out was this: if the piece of metal loses 
[a] due to melting, then the metal loses [a] because of a real change; after all, the melting of a piece of metal is a real change of it, and hence [a] is intrinsic to the metal. But if the piece of metal loses [a] due to a change of the law, then the piece of metal undergoes a mere Cambridge change which means that [a] comes out as extrinsic.

However, we see nothing special about our examples and suspect that for most properties it will be possible to generate analogous contradictory results, provided that one is imaginative enough. This is a problem because it means that Real Change* will typically yield the result extrinsic in cases where the intuitive result is intrinsic.

To convince ourselves of this, let us consider one more example property, which is intuitively intrinsic. In particular, let's consider the property we removed from the coin, which generated both a loss of [a] and a real change: the property of being solid. Surely, any way of removing the solidity of the coin will yield a real change in the coin. In that case, Real Change gives a determinate and intuitively satisfying result: solidity is intrinsic.

With a little imagination, however, we can remove the coin's solidity, but yield the intuition that the coin has not undergone a real change. Let's first get clear on the concept of solidity. A solid is often defined as a rigid material which does not flow when it is subjected to moderate forces (Doremus 1994: 339). Here 'moderate' is the important notion. Take a Maxwell body, such as glass. Glass flows like a fluid for small forces over long timescales (e.g. gravitational effect on window panes), is solid for small forces over short timescales (e.g. knuckle tap), and is brittle for large forces over short timescales (e.g. throwing bottles). Now consider a continental plate, which is solid when we walk on it (small force, small timescale), elastic in response to an Earthquake (large force, medium timescale), brittle in response to an asteroid (large force, small timescale), but fluid on the timescale of the age of Earth.

With this in mind, let's remove the solidity of the coin without really changing the coin, i.e., by only changing properties of objects that are wholly distinct from the coin. We simply change them in such a way so that what counts as 'moderate forces' changes: the dynamics of other objects relative to the coin are slowed right down so that from the perspective of the coin, only small forces are applied to the coin over large time-scales. But from the perspective of all other objects, these forces and time-scales are now considered the norm. For example, after holding the coin in your hand only over what you would (in this hypothetical scenario) consider a moderate time-scale, the coin, in response to gravity (moderate force), will ooze out across your palm into a puddle. No real change in the coin (at least prior to these gravitational effects), only a change that can be compared with Socrates when he becomes shorter than Theaetetus due to the latter's growth. But we have a loss of solidity, so by Real Change*, solidity is extrinsic. So for any putative intrinsic property of an object, just think of a possible world (however outlandish) in which the property is lost (or gained) simply by changing the states of other objects. In doing so Real Change* will always yield extrinsic.

The mistake made by Real Change (and Real Change*), we diagnose, is that it gets things the wrong way around. It seems that what it is for an object to undergo a real change (if this is meaningful notion at all) is for the object to lose an intrinsic 
property. And so to test for a real change we need to look for a change in intrinsic properties. But for this we need an independent test for intrinsicness.

\section{Perfect Duplication* and Lonely salvaged?}

Our progress on embarrassment reduction can be represented in the following (now depleted) table:

$\begin{array}{cll}\begin{array}{c}\text { [a] Being a one- } \\ \text { euro coin }\end{array} & \begin{array}{l}\text { Perfect Duplication* } \\ \text { Extrinsic }\end{array} & \begin{array}{l}\text { Lonely } \\ \text { Extrinsic }\end{array} \\ \begin{array}{c}\text { [b] Being in } \\ \text { Amsterdam }\end{array} & \text { Extrinsic } & \text { Extrinsic } \\ \begin{array}{c}\text { [c] Wearing a } \\ \text { golden ring }\end{array} & \begin{array}{c}\text { Depends on what's fed into the } \\ \text { machine }\end{array} & \begin{array}{c}\text { Depends on what's made } \\ \text { lonely }\end{array}\end{array}$

What about property [c], wearing a golden ring? Recall that Perfect Duplication failed to yield a result since it left open whether we should remove the ring before duplicating the Queen. This problem is not solved by Perfect Duplication* since this problem concerns what we feed into the machine, not where we locate the machine. And recall that Lonely applied to [c] failed to yield a result for the same reason. Perhaps the solution (for both Perfect Duplication* on [c] and Lonely on [c]) is simply to get clear on the referent of 'Queen': what are the objects that compose the Queen? Thus, we first make a judgment about composition, and then we apply the tests.

Naturally, we would not take the ring to be a component part of the Queen, and so we would remove the ring before the duplicator or the lonely world even come into play. If this is a legitimate response then we are finally having some success; for both tests now (apparently) give determinate and intuitively correct results.

Should we conclude that Perfect Duplication* and Lonely are adequate tests for intrinsicality? We think not. The relevant thought experiments now require that we isolate the ultimate component parts of the Queen. For Perfect Duplication*, we must isolate the relevant constituents so that we know what to put into the duplicator. For Lonely, we must isolate the relevant constituents so that we know what objects are to be made lonely. The problem is that unless we first understand the atomic constituents of the Queen, we have no clue as to what properties the Queen will retain if made lonely, or what properties the Queen's duplicate will retain. The final section is dedicated to pressing this problem.

\section{Farewell Lonely and Perfect Duplication*}

Our remaining tests, as we will now argue, are infected by a problem analogous to Hempel's famous dilemma for materialism (Hempel 1969). Materialism (roughly) states that everything is (or is made of, or is grounded in) matter. Materialism's 
dilemma is that 'matter' is either defined by present physics or by ideal future physics. If the former, materialism is (most likely) false since our present theories of matter are (most likely) false. If the latter then we don't know what materialism says because we don't know what ideal future physics says. This dilemma is relevant for Lonely and Perfect Duplication*. For when performing the relevant thought experiments we need to isolate the atomic constituents of the objects we are testing for the presence of intrinsic properties, before sending those objects (or their duplicates) into other possible worlds. The first step-isolating the atomic constituents-will require some understanding of those constituents. We either use our current limited understanding of the atomic constituents, or we use nonexistent (ideal completed) physics. Either way, we have no reliable way of executing the tests for a given case, as we will now argue.

The second horn of the dilemma is clearly problematic. Our tests are intended to offer ways of determining whether a given property is intrinsic or not. However, if the tests require a physical theory we do not have (ideal future physics) then we simply cannot perform the tests.

The first horn of the dilemma states that if the tests require current physics, then we cannot trust our tests due to the fallibility of the physical theories. To illustrate the fallibility of physics compare the following two theories, one that resembles classical physics, and one that resembles modern physics. We now show that even for the most mundane properties, test results are radically different depending on the physical theory one adopts. Here are the two theories:

Theory 1: Billiardballism: the atomic constituents of the Queen (and every other object) are tiny billiard balls, that differ from normal billiard balls only in scale-both ultimately obey the laws of Newtonian physics.

Theory 2: Global Nonseparability: the atomic constituents of the Queen (and every other object) are described by textbook quantum mechanics and the physical states (including the positions) of any given atom in the universe are nonseparable from the physical states of all other atoms in the universe. ${ }^{7}$

Billiardballism is self-explanatory. Let's begin by considering how Lonely categorizes the Queen's shape, and the Queen's wearing a golden ring, given Billiardballism. We take all the atoms that compose the Queen, and remove every other atom, to see whether the Queen retains her shape and her ring. In the lonely universe the atomic billiard balls retain their configuration. Since the Queen's shape presumably supervenes on the configuration of her atomic parts, the Queen presumably retains her shape. Her shape is therefore intrinsic. ${ }^{8}$ Since we do not

\footnotetext{
7 We define textbook quantum mechanics as a realist theory that describes physical systems in terms of Hilbert space formalism. Nonseparability is defined by Esfeld (2004) as follows: "The states of two or more systems are nonseparable iff it is only the joint state of the whole that completely determines the state-dependent properties of each system and the correlations among these systems (to the extent that these are determined at all)".

${ }^{8}$ One might worry that the sudden loss of surrounding objects will gravitationally affect the shape of the Queen, but the point of the thought experiment, we take it, is to consider the Queen at the first moment of loneliness, before the effects of having no surroundings come into play.
} 
deem the atomic parts of the Queen's ring to be parts of the Queen, the ring's parts are removed, so the Queen does not retain the ring. Her wearing a golden ring is therefore extrinsic.

Perfect Duplication*, given Billiardballism, will effectively give the same results: since the configuration of the Queen's parts are duplicated, her shape is duplicated (necessarily), so her shape is intrinsic; since the ring's parts are not duplicated, her ring is not duplicated (necessarily), so her wearing the ring is extrinsic.

Before we consider how Global Nonseparability affects our tests, a gentle introduction to the idea of nonseparability will be useful. To simplify, consider an atom that is a part of the Queen (particle 1) and another that is not part of the Queen (particle 2). In quantum mechanics, particle states (e.g. particle positions) are designated by vectors. So if particle 1 is located at $X a$ (i.e. point $a$ on the $X$ axis) then we represent its position with the term $\mid X a>_{1}$, where ' $\mid>$ ' indicates a vector. However, particles are not typically in definite positions (the same goes for other physical properties like momentum and spin). Instead, particles are in so-called superpositions of different positions. Metaphysically, superpositions are notoriously difficult. But mathematically they are well understood, and can be represented by weighted sums of vectors. For example, particle 1's being in a superposition of Xa and $\mathrm{Xc}$ is represented as $\#\left|\mathrm{Xa}>{ }_{1}+\#\right| \mathrm{Xc}>_{1}{ }^{9}$ Crucially, if particle 1 has (in almost any way) interacted with particle 2 , then their positions will be nonseparable such that their joint position-state will be represented by something like:

$$
\#\left(\left|X a>_{1}\right| X b>_{2}\right)+\#\left(\left|X c>_{1}\right| X d>_{2}\right)
$$

According to this quantum state, particle 1 is not (strictly speaking) in a superposition of being located at Xa and being located at Xc: it is in a nonseparable superposition with particle 2. This entails that particle 1 has no position of its own: the nonseparable state is (as the name suggests) not decomposable into individual component position states. Global nonseparability is the thesis that no particle has a position of its own: every particle is in a nonseparable superposition with every other particle.

Now consider what happens when we try to make the Queen's constituents lonely, given Global Nonseparability. Since the positions of 1 and 2 are nonseparable, if we remove particle 2 we inevitably remove particle 1 . The idea is that removing particle 2 entails that particle 2 no longer has a position. But removing 2's position inevitably removes particle 1's position, thereby removing particle 1 itself. Given global nonseparability, particle 2's position is nonseparable with all the particles that compose the Queen. So removing particle 2 removes the

\footnotetext{
$9 \mathrm{IXa}>$ and $\mathrm{IXc}>$ will be orthogonal unit-length vectors in a high-dimensional vector space. For an easy introduction to this formalism see Albert (1992: Chapter 2). Mathematically, our example involves a superposition of delta functions (eigenfunctions of the position operator). Such functions are problematic in quantum mechanics and are used here just for simplicity. Our argument could equally well be expressed in terms of superpositions of Gaussian functions.
} 
Queen. ${ }^{10}$ Similarly for the Queen's property of wearing a golden ring: since the positions of the ring's constituents and the positions of the Queen's constituents are nonseparable, removing the ring (i.e. literally removing it from the world in the process of making the Queen lonely) removes the Queen. This would appear to destroy the test entirely: given Global Nonseparability, it is simply not possible to make the Queen lonely.

Assuming Global Nonseparabilty, is there any way to recast Lonely in such a way that it can yield determinate results? We've been working with a conception of Lonely under which one removes all particles except for those that constitute the property bearer of interest. But we could try to make the property bearer lonely in another way, viz. by transporting it to an empty possible world. But even that wouldn't help: how could particle 1 be transported to another world by itself, given its nonseparability with particle 2? Perhaps, however, we can fix Lonely with an additional ad hoc stipulation: if particles to be made lonely happen to be nonseparable from particles that are not to be made lonely, then "separate" them first. However, since separating nonseparable particles requires changing their physical states entirely, the question arises: what states do we change them to before making them lonely? The states that guarantee that Lonely yields intuitive results? But this would make Lonely circular. Furthermore, even if we fixed on some way of separating nonseparable particles, we have no reliable way of knowing what effect that will have on a complex object like the Queen, so we would not know what properties she would retain, so Lonely still gives no results.

Finally, one might think that if it really is the case that removing particle 2 removes the Queen, then this is evidence that particle 2 is actually a part of the Queen after all. But then given global nonseparability, the Queen is composed of every particle in the universe. Removing every particle that does not compose the Queen then means removing nothing. Alternatively, transporting the Queen to an empty world means duplicating the actual world. Consequently, every property of the Queen would turn out to be intrinsic. This is the bullet that we think defenders of Lonely must ultimately bite. Before explaining the problem with this consequence, we will show that the same problem arises for Perfect Duplication*.

As with Lonely there are two ways we can understand the duplication test: either the duplication machine puts the duplicate in the actual world (Perfect Duplication) or it puts the duplicate in any possible world (Perfect Duplication*). Either way, the problem is that we cannot literally isolate the Queen's parts to put them (and only them) in the machine, given their nonseparability with all other particles. Duplicating the Queen in the actual world (in accordance with Perfect Duplication) requires that the Queen-duplicate is constructed so that her parts are nonseparable from every other particle in the universe in the same way as the Queen-original. We cannot see any way of making sense of this situation. Alternatively, if the machine puts the Queen in an alternative possible world, then to be a duplicate her nonseparable state must remain, and so the machine does not duplicate only the

\footnotetext{
${ }^{10}$ Strictly speaking what's removed is the Queen's property of having a position (any position). One might maintain that something is nonetheless made lonely: a position less bare particular-but such an entity would not be the Queen and would not retain any interesting properties.
} 
Queen, but duplicates the entire actual world in the alternative possible world. Here we at least get meaningful results, but ones which deem all of the Queen's properties intrinsic. This is the bullet that we think defenders of Perfect Duplication* must ultimately bite.

We now have our illustration of (horn one of) our Hempel-like dilemma: Lonely and Perfect Duplication* give radically different results depending on whether we appeal to a theory resembling classical physics (Billiardballism) or a theory resembling modern physics (Global Nonseparability). For example, on the former theory, only a small portion of the Queen's properties are intrinsic, while on the latter, all of them are.

Now, a defender of these tests might simply bite these bullets and respond that although the tests do not give us the information we were hoping for, they still give us some information-enough to render them acceptable tests for intrinsicality. In particular, although the tests do not yield information of the form:

properties $\mathrm{X}, \mathrm{Y}$, and $\mathrm{Z}$, are intrinsic;

properties $\mathrm{U}, \mathrm{V}$, and $\mathrm{W}$ are non-intrinsic;

they at least yield information of the form:

if GNS then properties $\mathrm{X}, \mathrm{Y}$, and $\mathrm{Z}$, are intrinsic;

if Billiardballism then properties $\mathrm{X}, \mathrm{Y}$, and $\mathrm{Z}$ are non-intrinsic.

However, we do not think these conditionals are enough to render them acceptable tests. To explain why we use an analogy. Suppose a physician knows the following two conditionals:

if my patient $\mathrm{S}$ suffers from disease $\mathrm{D}$, then cure $\mathrm{C}$ will cure $\mathrm{S}$;

if $\mathrm{S}$ suffers from disease $\mathrm{D}^{*}$, then $\mathrm{C}$ will kill $\mathrm{S}$;

but the medical tests available to him don't enable him to conclude whether S suffers from $\mathrm{D}$ or $\mathrm{D}^{*}$. Then these conditionals, true as they may be, are not epistemic helps for deciding whether or not to apply cure C. Analogously, the intrinsicality conditionals, true as they may be, are not epistemic helps for deciding whether or not to apply the predicate 'is intrinsic'.

Is there any way other to salvage our tests? One common reaction we have faced by defenders of intrinsicness consists in the following suspicion: our argument somehow depends on the assumption that worlds containing lonely individuals (or worlds that host the duplicates) are governed by the same laws of nature as the actual world. The upshot is then meant to be that the interpretation of quantum mechanics is irrelevant to what happens in such worlds. ${ }^{11}$ However, this reaction is based on a fundamental misunderstanding of the nature of quantum nonseparabilty.

The reaction appears to suppose that by placing the Queen in a world with different laws (or no laws at all), the nonseparabilty of the Queen's components somehow vanishes. But this is wrong. Imagine that we aim to make the Queen

\footnotetext{
${ }^{11}$ Note that Vallentyne $(1997,212)$, in his discussion of Lonely, requires that laws of nature are to be removed from the relevant world, as well as all contingent objects other than the one possessing the putative intrinsic property.
} 
lonely. Will removing the laws of quantum mechanics remove the nonseparabilty of the Queen's components? No it will not. The reason is that nonseparabilty refers not to features of the laws of quantum mechanics, but to physical states that are brought on by the laws of quantum mechanics. If you remove the laws of nature you do not thereby remove the physical states that those laws previously gave rise to. This reaction therefore appears to treat nonseparability as a feature of physical laws when in reality it is a feature of physical states.

Perhaps what the defender of intrinsicality wants is this: when the Queen is duplicated to a possible world with different (or no) laws, or when she is made lonely (in a way that removes quantum laws) then any physical states that can only be brought on by actual-world-laws must be removed. But this is no help either: how do we go about removing the relevant physical states of the Queen (or her duplicate)? As mentioned above, doing so will inevitably change the Queen. But there is no fact of the matter as to how this will change her! The defender of intrinsicality may want to change the Queen so that she is gauranteed to retain only those properties that are intuitively intrinsic. But then the test itself becomes superfluous, and we are left with no reliable way of testing for intrinsicness.

Are there any other ways out for the defender of intrinsicality? Perhaps one could reject Global Nonseparability in some way. Let's consider two responses along these lines. The first denies modern quantum mechanics in general, the second denies Global Nonseparability more specifically.

One might respond with skepticism about modern physics, and therefore skepticism about any theory that resembles it, such as Global Nonseparabilty. Indeed, a response in this spirit was advocated by David Lewis when he realized that an aspect of his metaphysics made the same problematic assumption that is apparently made by Lonely and Perfect Duplication. The assumption often comes under the label locality. To the nonlocality ${ }^{12}$ inherent in quantum mechanics Lewis famously replied:

I am not ready to take lessons in ontology from quantum physics as it is now. First I must see how it looks when it is purified of instrumentalist frivolity, and dares to say something not just about pointer readings but about the constitution of the world; and when it is purified of double thinking deviant logic; and-most of all-when it is purified of supernatural tales about the observant mind to make things jump. If, after all that, it still teaches nonlocality, I shall submit willingly to the best of authority. (Lewis 1986: xi)

Could a similar defense be given on behalf of our tests? That is, could we ignore the results from above by asserting that quantum mechanics itself is in bad shape (e.g. due to the suspicious causal role it apparently attributes to the observant mind)? Not likely, since modern realist materialist reconstructions of quantum mechanics still

\footnotetext{
12 Nonlocality is an implication of nonseparability. Some interactions with nonseparable particles are nonlocal in the sense that effecting a change on particle 1 in region A can instantaneously influence how particle 2 will react to an interaction in region B, where A and B are arbitrarily far apart (Albert 1992: Chapter 3).
} 
postulate nonlocality. ${ }^{13}$ Furthermore Global Nonseparability itself is hardly an outlandish possibility. As Schaffer (2010 Sect. 2.2) has pointed out, many physicists suspect that the big bang made the positions of all particles nonseparable. Thus, if anything, Global Nonseparability should be a serious candidate physical theory that guides our tests.

This brings us to the second response: instead of denying quantum mechanics, just deny Global Nonseparabilty, and advocate an alternative understanding of quantum mechanics. There is some precedent for this. After all, quantum mechanics faces difficult problems, which have given rise to a number of so-called "interpretations" of quantum mechanics, which are essentially different physical theories attempting to deal with those problems. And in particular, some interpretations introduce additional fundamental ontology over and above what is described by Global Nonseparability (e.g. Bohm 1952). Perhaps it could be argued that such additions (and their composites) have some intrinsic properties that our tests classify in an intuitively satisfying way. However, this simply brings out how sharp the first horn of the dilemma is: there is no consensus at all as to which (if any) is the correct interpretation of quantum mechanics. Thus, for even the most mundane properties (such as the Queen's wearing a golden ring), our tests only have a chance of giving intuitive results under very specific, highly controversial, interpretations of quantum mechanics. The fact that intuitive results may only obtain under theories resembling classical physics suggests that intrinsicality may just be a notion that belongs to a metaphysics that builds on a now outdated physics. At any rate, we conclude that the tests for intrinsicness considered here cannot be relied upon.

Acknowledgments For comments on and discussion of earlier versions of this paper we are indebted to: Robert Audi, Arianna Betti, Dan Marshall, Fred Muller, Rik Peels, Jeroen de Ridder, and Emanuel Rutten. It is gratefully acknowledged that work on this paper was made possible by a grant from the Templeton World Charity Foundation. The views expressed in this paper are those of the authors.

Open Access This article is distributed under the terms of the Creative Commons Attribution 4.0 International License (http://creativecommons.org/licenses/by/4.0/), which permits unrestricted use, distribution, and reproduction in any medium, provided you give appropriate credit to the original author(s) and the source, provide a link to the Creative Commons license, and indicate if changes were made.

\section{References}

Albert, D. Z. (1992). Quantum mechanics and experience. Massachusetts: Harvard University Press. Bohm, D. (1952). A suggested interpretation of the quantum theory in terms of "Hidden" variables, I and II. Physical Review, 85, 166-193.

\footnotetext{
13 For example, dynamical collapse theories, whose collapse functions do not actually yield separability (McQueen 2015: Sect. 2). Furthermore, the relevant aspect of Lewis' metaphysics is called Humean Supervenience and few "Humeans" follow Lewis in his hope that physics might someday restore locality. A natural solution for Humeans simply treats nonseparability as a new kind of fundamental relation over and above spatiotemporal relations (Darby 2012). While this may save (something similar to) Humean Supervenience, it does not help our tests at all, since removing such relations still removes the positions of the particles to be made lonely.
} 
Darby, G. (2012). Relational holism and humean supervenience. British Journal for the Philosophy of Science, 63(4), 773-788.

Doremus, R. H. (1994). Glass science (2nd ed.). New York: Wiley.

Esfeld, M. (2004). Quantum entanglement and a metaphysics of relations. Studies in the History and Philosophy of Modern Physics, 35(4), 601-617.

Figdor, C. (2008). Intrinsically/Extrinsically. Journal of Philosophy, 105, 691-718.

Francescotti, R. (1999). Mere cambridge properties. American Philosophical Quarterly, 36, $295-308$.

Geach, P. (1969). God and the soul. Bristol: Thoemmes.

Hempel, C. G. (1969). Reduction: Ontological and linguistic facets. In S. Morgenbesser, P. Suppes, \& M. White (Eds.), Philosophy, science, and method: Essays in honor of Ernest nagel (pp. 179-199). New York: St. Martin's Press.

Lewis, D. K. (1983). "Extrinsic Properties". In Lewis 1999: 111-115.

Lewis, D. K. (1986). Philosophical papers (Vol. 2). Oxford: Oxford University Press.

Marshall, D. (2013). Analyses of intrinsicality without naturalness. Philosophy Compass, 8(2), $186-197$.

McQueen, K. J. (2015). Four tails problems for dynamical collapse theories. Studies in the History and Philosophy of Modern Physics, 49, 10-18.

Schaffer, J. (2010). Monism: The priority of the whole. Philosophical Review, 119(1), 31-76.

Searle, J. (1995). The construction of social reality. New York: The Free Press.

Sider, T. (1996). Intrinsic properties. Philosophical Studies, 83(1996), 1-27.

Stump, E., \& Kretzman, N. (1985). Absolute simplicity. Faith and Philosophy, 2(1985), 353-391.

Vallentyne, P. (1997). Intrinsic properties defined. Philosophical Studies, 88, 209-219.

van Inwagen, P. (2009). Metaphysics (3rd ed.). Boulder: Westview Press.

Weatherson, B. \& Marshall, D. (2013). "Intrinsic vs. Extrinsic Properties", The Stanford Encyclopedia of Philosophy (Spring 2013 Edition), Edward N. Zalta (ed.); URL: http://plato.stanford.edu/archives/ spr2013/entries/intrinsic-extrinsic/. 